\title{
Un caso teratológico en un curculiónido (Coleoptera: Curculionoidea) de la Región de Magallanes (Chile)
}

\author{
A teratological case in a curculionid beetle \\ (Coleoptera: Curculionoidea) from Magallanes Region (Chile)
}

Eduardo I. Faúndez ${ }^{1,2} \&$ Mariom A. Carvajal1,2

\section{Resumen}

Se describe un caso teratológico de Aegorhinus vitulus (Fabricius, 1755) recolectado, al estado adulto, en la Región de Magallanes. La anomalía corresponde a una hemiteria, y dentro de éstas, específicamente a una displasia elitral bilateral. Se estima que la causa de esta malformación podría tener un origen exógeno, producido por alguna presión externa durante la fase de pupa del insecto. El presente caso es el tercero descrito para una especie de Curculionidae en Chile y el segundo para $A$. vitulus.

\section{Palabras clave:}

Teratología, hemiteria, displasia, Aegorhinus vitulus.

\begin{abstract}
A teratological case is described for the weevil Aegorhinus vitulus (Fabricius, 1755). The adult specimen was collected in Magallanes Region. This abnormality belongs to the group of the hemiteries. Within this teratosis it specifically is a bilateral elytral dysplasia. It is estimated that the malformation may has an exogenous origin. This produced because of an external influence during pupal stage. This case is the third recorded for a curculionid beetle in Chile, but also the second for $A$. vitulus.
\end{abstract}

\section{Key words:}

Teratology, hemiterie, dysplasia, Aegorhinus vitulus.

\section{INTRODUCCIÓN}

La teratología comprende el estudio de las malformaciones, deformaciones y ejemplares estropeados (i.e. en el caso de los artrópodos) (Dallas, 1926; Pérez D’Angello, 1967). Las teratosis son sucesos singulares que acaecen en los individuos incidiendo en su ontogenia, pero en ocasiones pueden perdurar en el tiempo y manifestarse en la filogenia de un determinado linaje (Ortuño \& Ramos, 2008). A nivel mundial, los insectos del orden Coleoptera son los que registran la mayor cantidad de reportes (Balazuc, 1948, 1969). En Chile la situación no es distinta, ya que este grupo es ampliamente el que registra la mayor cantidad de reportes (Pérez D’Angello, 1967), situación que se mantiene hasta el día de hoy (Lüer, 2015). De éstos, solo existen dos casos descritos para especies de la familia Curculionidae, uno para Aegorhinus superciliosus (Guérin-Méneville, 1830) (Aguilera et al. 2007) y otro para Aegorhinus vitulus (Fabricius, 1755) (Faúndez, 2007). Aegorhinus vitulus, comúnmente conocido como caballito de palo o caballito del monte, es una de las especies más características del extremo sur de Patagonia, se le encuentra asociado a bosques de Nothofagus Blume (Nothofagaceae) (Faúndez, 2007), y se

1 Entomology Department, School of Natural Resource Sciences, North Dakota State University, Fargo, ND, USA. $\triangle$ ed.faundez@gmail.com.

2 Departamento de Zoología Médica, Centro de Estudios en Biodiversidad (CEBCh), Magallanes, 1979, Osorno, Chile. 
ha indicado que era un insecto sagrado para los indígenas de Tierra del Fuego (Dimitri, 2015). La presente contribución tiene por objeto describir un nuevo caso teratológico para esta especie.

\section{MATERIALES Y MÉTODOS}

En terminología se sigue a Dallas (1926) y Balazuc (1948, 1969). Las fotografías fueron tomadas con una cámara digital de alta resolución (Kodak ${ }^{\circledR}$ C550).

Material examinado: Chile, Región de Magallanes, Punta Arenas, Parque María Behety, 20-10-2012, Faúndez \& Carvajal leg., 1今, En Nothofagus antarctica (Forster) Oerst. El ejemplar se mantuvo vivo en condiciones de laboratorio, alimentado con follaje de $N$. antarctica, por siete semanas (El ejemplar se encuentra depositado en la colección del Centro de Estudios en Biodiversidad CEBCh).

\section{RESULTADOS}

El presente caso corresponde al grupo de las hemiterias, teratosis definidas como desviaciones ligeras, que incluyen deformaciones, malformaciones, estropeados y variaciones diversas (Dallas, 1926). Dentro de éstas, específicamente corresponde a una displasia elitral bilateral, la que es descrita a continuación:

Displasia elitral bilateral en Aegorhinus vitulus (Figs. 1, 2)

El ejemplar presenta una deformación en la sección distal de ambos élitros (Fig. 1). Esta deformación consiste en un leve acortamiento de las caras internas de ambos élitros, la que es levemente más pronunciada en el élitro izquierdo. Debido a esta situación ambos élitros no logran reunirse en la sección terminal del abdomen del insecto (Fig. 2), dejando al descubierto el segmento terminal del abdomen; mientras que en los casos normales este se encuentra cerrado completamente por los élitros formando un caparazón. Los bordes internos de ambos élitros se observan normales, y presentan una pequeña carena longitudinal que no evidencia ningún golpe o quiebre por presión externa.

Comentario: La pata mesotorácica derecha

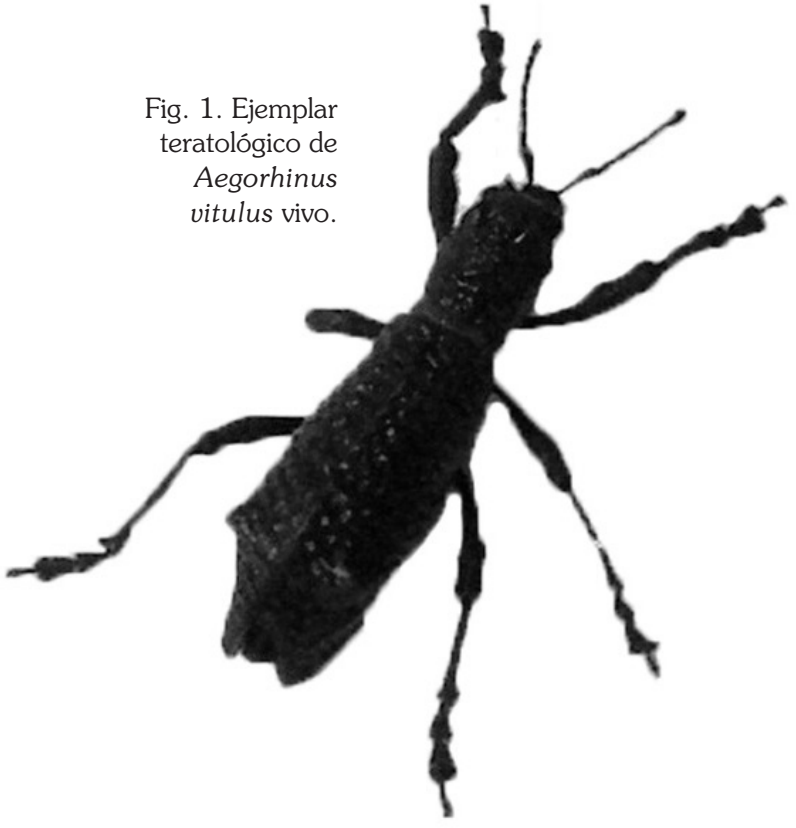

Fig. 2. Ejemplar teratológico de Aegorhinus vitulus, detalle de la displasia elitral.

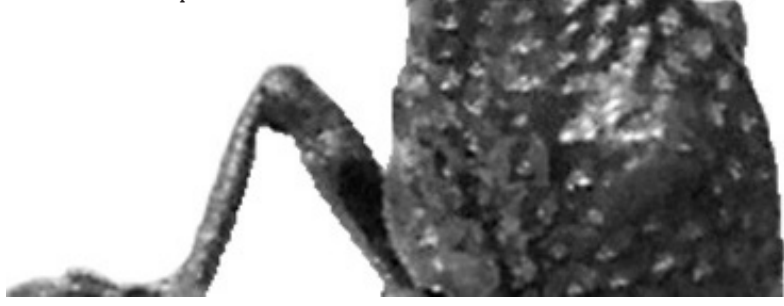

del ejemplar se encuentra mutilada (Fig. 1), no obstante esto corresponde a una herida sufrida en estado adulto (probablemente por el ataque de algún predador), y no tiene relación con agentes teratológicos.

\section{DISCUSIÓN Y CONCLUSIONES}

El presente caso es similar al descrito por Faúndez (2007), quien también reporta una displasia elitral. Sin embargo el ejemplar descrito en ese trabajo, la diferencia entre un élitro y el otro es mucho más notoria, siendo unilateral, con el élitro derecho normal. Adicionalmente en ese caso el élitro izquierdo evidencia una terminación falciforme. En este reporte, ambos élitros presentan una terminación recta, siendo poco probable que ambas malformaciones (i.e. Faúndez, 
2007 y la aquí descrita) se deban exactamente a las mismas causas. En este caso, es probable que la malformación tenga algún origen exógeno, debido a alguna presión durante el estado de pupa. Lo que indujo a algún desajuste morfológico impidiendo la correcta formación de la sección terminal de los élitros. Esta situación suele ser la causa más común para este tipo de malformación en coleópteros (Balazuc, 1948). Es interesante mencionar que esta malformación aparentemente no afectó en mayor medida al ejemplar, que fue recolectado como adulto maduro en terreno, sin que ésta otorgara dificultad alguna al insecto. Adicionalmente el tiempo de crianza transcurrido en condiciones de laboratorio corrobora esta idea, estimándose que el insecto murió de forma, aparentemente, natural. Curculionidae es una de las familias con menor cantidad de casos registrados en el país (Pérez D’Angello, 1967; Faúndez, 2007); convirtiéndose este caso, en el tercero formalmente descrito, y en el segundo correspondiente a una anomalía elitral.

\section{LITERATURA CITADA}

Aguilera, A., Klein, C., \& Rebolledo, R. (2007). Larva bicéfala de Aegorhinus superciliosus (Guérin) (Coleoptera: Curculionidae). Revista Chilena de Entomología, 32, 41-43.

Balazuc, J. (1948). La tératologie de Coléoptères, et expériencesde transplantation chez Tenebrio molitor L. Memoires Du Museum National D'Historie Naturelle (Paris), 25, 1-293.

Balazuc, J. (1969). Supplément à la Tératologie des Coléoptères. Redia, 51(3), 39-111.

Dallas, E .D. (1926). Anomalías en coleópteros chilenos. Revista Chilena de Historia Natural, 30, 73-83.

Dimitri, M. J. (2015). Fagáceas del bosque Patagónico. Del escritorio al campo, 2, 3pp. http://awsassets.wwfar.panda.org/ downloads/escritorio_al_campo___rev_ vs_80___arboles_del_bosque_andino_ patagonico.pdf (acceso 07/12/2015).

Faúndez E. I. (2007). Descripción de un caso teratológico en Aegorhinus vitulus (Fabricius, 1775) (Coleoptera: Curculionidae) de la Región de Magallanes (Chile). Boletín de la Sociedad Entomológica Aragonesa, 40, 470.

Lüer, A. (2015). Un caso teratológico en Eucaliga sanguinicollis Fairmaire \& Germain, 1861 (Coleoptera: Tenebrionidae). Biodiversity and Natural History, 1(2), 41-43.

Ortuño, V. M. \& Ramos Abuin, J. A. (2008). Reflexiones sobre la teratología y descripciones de cuatro teratosis apendiculares en Coleoptera. Boletín de la Sociedad Entomológica Aragonesa, 43, 435-439.

Pérez D’Angello, V. (1967). Contribución a la entomoteratología. Noticiario Mensual del Museo Natural de Historia Natural (Santiago, Chile), 11, 4pp. 
